\title{
Description of all conformally invariant differential operators, acting on scalar functions
}

\author{
Petko Nikolov $\dagger$, Tihomir Valchev $\ddagger$ \\ $\dagger$ Department of Theoretical Physics, Sofia University, \\ 5, James Bourchier Blvd., 1164 Sofia, Bulgaria \\ $\ddagger$ Institute for Nuclear Research and Nuclear Energy,Bulgarian Academy of \\ Sciences, 72 Tzarigradsko chaussè̀, 1784 Sofia, Bulgaria
}

\begin{abstract}
We give an algorithm write down all conformally invariant differential operators acting between scalar functions on Minkowski space. All operators of order $k$ are nonlinear and are functions on a finite family of functionally independent invariant operators of order up to $k$. The independent differential operators of second order are three and we give an explicit realization of them. The applied technique is based on the jet bundle formalism, algebraization of the the differential operators, group action and dimensional reduction. As an illustration of this method we consider the simpler case of differential operators between analytic functions invariant under the modular group. We give a power series generating explicitly all the functionally independent invariant operators of an arbitrary order.
\end{abstract}

\section{Introduction}

It is well-known that the Maxwell equations are conformally invariant. This motivate an permanent interest in studying the conformal classes of metrics, conformally invariant operators and structures. The Maxwell differential operator is linear and so it is a splitting operator between two (infinitely dimensional) linear representation of the conformal group. There are many papers in the literature treating the splitting operators between different representations of the conformal group. These operators generalize the Maxwell operator with respect of this property of invariance. These considerations are based on the studying of the description and the structure of the linear representations and their subrepresentations.

The aproach applied in the present work is different. It is based on jet bundles technique (3]), ([5]) and ([6]). The jet lifting of functions (or sections) plays a role of an universal differential operator. The differential operators are viewed as a composition of a jet lifting and a fibre preserving map (a morphism for the case of linear operators) between vector bundles. This algebraise the differential operators and after this the invariance means invariance of these maps (morphisms). During the next step the technique of group action and dimensional reduction are used to describe the invariant fibre-preserving maps (sections). A crucial point is the study of the action of stationary subgroup of some point on the jets of smooth sections at this point. This contains elements of the Catastrophe theory in the sense of R. Tom and Arnold (see [1] and [2]). If the steps have been done explicitly this prescription gives all invariant operators including the nonlinear ones.

In our case (differential operators acting on scalar functions on Minkowski space) the main result is the following: all invariant operators are nonlinear of order equal or bigger than two (we exclude the trivial case of zero order operators). For second order $(n=2)$ there are three independent differential operators (see 35, 36 and 37). Any other invariant operator of order two is a function of three variables of them. ASimilarly, for order $n=3$ there are 23 independent operators - the previous three and 20 operators more of order three. Every invariant operator up to order three is a function of these universal operators. For the case of an arbitrary finite order 
$n$ there exist a finite complete system of independent conformally invariant operators up to order $n$ i.e. they generate all other invariant operators up to the corresponding order.

In the simpler case of differential operators between analytic functions, invariant under the modular group the result is similar. All invariant operators are nonlinear. There are $n-2$ independent operators of the order up to $n$. All others of are functions of them. We give a power series generating the family of independent invariant differential operators. The invariant operator of order three is closely related to the Schwarz derivative (there is a difference because the Schwarz derivative takes values in the quadratic differentials).

\section{General Scheme}

Let $\xi=(E, p . M)$ be a vector bundle where $E$ is a $m+n$-dimensional manifold and $M$ is a $m$ dimensional manifold. Smooth sections $M \rightarrow E$ build an infinite dimensional space denoted by $C^{\infty}(\xi)$. We will often use adapted coordinates $\left(x^{\mu}, u^{a}\right)$, i.e. coordinates satisfying the relation $p\left(x^{\mu}, u^{a}\right)=\left(x^{\mu}\right), \mu=1, \ldots, m, a=1, \ldots, n$.

A connected Lie group $G$ acts on $\xi$ by bundle morphisms

$$
\forall g \in G \exists T_{g} \in \operatorname{Diff}(E): T_{g}: \xi_{x} \rightarrow \xi_{t_{g}(x)},
$$

where $\xi_{x}=p^{-1}(x)$ is the fibre over $x \in M$ and $t_{g} \in \operatorname{Diff}(M)$ is the projection of a morphism $T_{g}$. In adapted coordinates $\left(x^{\mu}, u^{a}\right)$ the action of $G$ reads

$$
\left(x^{\mu}, u^{a}\right) \rightarrow\left(\left(t_{g}\right)^{\mu}(x),\left(T_{g}\right)_{b}^{a}(x) u^{b}(x)\right) .
$$

The group $G$ has a natural action on $C^{\infty}(\xi)$

$$
\begin{gathered}
G \times C^{\infty}(\xi) \ni(g, \psi) \longrightarrow g(\psi) \in C^{\infty}(\xi) . \\
g(\psi)(x):=T_{g}\left(\psi\left(t_{g}^{-1}(x)\right)\right) .
\end{gathered}
$$

In the local coordinates $\left(x^{\mu}, u^{a}\right)$ this action is given by

$$
g(\psi)^{a}(x)=\left(T_{g}\right)_{b}^{a}\left(t_{g}^{-1}(x)\right) \psi^{b}\left(t_{g}^{-1}(x)\right) .
$$

A natural problem is the description of the vector subspace $C^{\infty}(\xi)_{G} \subset C^{\infty}(\xi)$ of all $G$-invariant sections. The invariance condition $g(\psi)=\psi$ locally looks as

$$
\left(T_{g}\right)_{b}^{a}\left(t_{g}^{-1}(x)\right) \psi^{b}\left(t_{g}^{-1}(x)\right)=\psi^{a}(x)
$$

or equivalently

$$
\left(T_{g}\right)_{b}^{a}(x) \psi^{b}(x)=\psi^{a}\left(t_{g}(x)\right) .
$$

In general a description of all $G$-invariant sections is hardly possible but under some natural requirements imposed on the group action it may be achieved. There is a "smaller" reduced bundle $\xi_{G}$ that smooth sections (without any restriction) in it are one-to-one correspondent with $G$-invariant sections in $\xi$ (elements of $\left.C^{\infty}(\xi)_{G}\right)$. The abstract algebraic construction of $\xi_{G}$ consists of two steps. Consider the stationary subgroup $H_{x_{0}}=\left\{h \in G \mid t_{h}\left(x_{0}\right)=x_{0}\right\}$ of a point $x_{0} \in M$. For $x=x_{0}$ the $G$-invariance condition (2) is

$$
\left(T_{h}\right)_{b}^{a}\left(x_{0}\right) \psi^{b}\left(x_{0}\right)=\psi^{a}\left(x_{0}\right), \quad h \in H_{x_{0}} .
$$

As a matter of fact, this is a restriction on the values of $G$-invariant sections at any given point $x_{0}$. Let $s t(\xi)_{x_{0}}=\left\{\vec{u} \in \xi_{x_{0}} \mid T_{h}(\vec{u})=\vec{u}, \forall h \in H_{x_{0}}\right\}$ be the vector subspace of all fixed vectors. We assume that the collection of all spaces $s t(\xi)_{x}$ for all $x \in M$ is a vector bundle $s t(\xi) \subset \xi$ called a stationary subbundle. This condition limits the action of $G$. Obviously, $C^{\infty}(\xi)_{G} \subset C^{\infty}(s t(\xi))$.

Note: The explicit construction of the stationary subbundle $s t(\xi)$ is the crucial point where the new structure of the reduced bundle arises. This is the most difficult step in our approach. 
The second step consists in taking the quotient of the base $M$ for the bundle $s t(\xi)$. We suppose that the projection $t_{g}$ has uniform orbits in the base $M$ and $M$ itself represents a total space of a smooth locally trivial bundle $(M, \pi, M / G)$ of homogeneous spaces. It is another requirement on the action of the group $G$. Let's consider an orbit of $G$ linking up $x, y \in M$, i. e. $\exists g \in G: y=t_{g}(x)$. If $\psi \in C^{\infty}(\xi)_{G}$ the value $\psi(y)$ is uniquely determined by the value of the section at $x$ (in accordance to the invariance condition (2)

$$
\psi^{a}(y)=\psi^{a}\left(t_{g}(x)\right)=\left(T_{g}\right)_{b}^{a}(x) \psi^{b}(x) .
$$

Let $(M, \pi, M / G)$ be a trivial bundle and $N$ be a global section i.e. $N \subset M$ is transversal to the orbits. Because of the relation (4), a $G$-invariant section $\psi$ is completely determined if we know the restriction $\left.\psi\right|_{N}\left(\left.\psi\right|_{N} \in C^{\infty}\left(\left.s t(\xi)\right|_{N}\right)\right)$. Moreover, if $\varphi \in C^{\infty}\left(\left.s t(\xi)\right|_{N}\right)$ then $\varphi$ correctly induces an invariant section $\psi \in C^{\infty}(\xi)_{G}$. Indeed, let $y \in M$, then the orbit through $y$ intersects the submanifold $N$ only in one point $x: y=t_{g}(x)$ for some $g \in G$. By definition, the induced $G$-invariant section is

$$
\psi^{a}(y):=\left(T_{g}\right)_{b}^{a}(x) \varphi^{b}(x) .
$$

The element $g \in G$ is not uniquely determined by $y$ and $x$ but since $\varphi(x) \in \operatorname{st}(\xi)_{x}$ the value $\psi(y)$ doesn't depend on the specific choice of the group element. The restriction $\left.s t(\xi)\right|_{N}$ is a coordinate realization of the bundle $\xi_{G}$. If we consider another submanifold $N^{\prime} \subset M$ transversal to the orbits in $M$, the corresponding restriction $\left.s t(\xi)\right|_{N^{\prime}}$ is another coordinate realization. There is a canonical isomorphism $\left.s t(\xi)_{N^{\prime}} \approx s t(\xi)\right) N$ induced by the group action. This procedure sews the abstract reduced bundle from the coordinate realization. If $(M, \pi, M / G)$ isn't trivial the construction of $\xi_{G}$ is analogous but slightly complicated. We have to sew local coordinate realizations. Smooth sections in the reduced bundle are one-to-one correspondent with $G$-invariant sections. We shall transform the problem of describing invariant differential operators into a problem for characterization of invariant sections in appropriate jet bundles.

Consider two bundles $\xi$ and $\eta$ over the same base $M$. A group $G$ acts on both of them by the same projection $t$ in $M$. The action of $G$ on $C^{\infty}(\xi)$ and $C^{\infty}(\eta)$ induces an action on differential operators $D: C^{\infty}(\xi) \rightarrow C^{\infty}(\eta)$

$$
g(D)(\psi):=g\left(D\left(g^{-1}(\psi)\right)\right) .
$$

We use for simplicity the same notation for the actions of $G$ on $\xi$ and on $\eta$. A differential operator is called $G$-invariant if it satisfies the following condition

$$
g(D)(\psi)=D(g(\psi)), \quad \forall g \in G, \forall \psi \in C^{\infty}(\xi) .
$$

The problem we consider is the description of all invariant differential operators. This problem can be reduced to the problem we studied before by using the jet bundle technique (for more details about jet bundles see [3, [4, [5], 6]). Let a differential operator $D: C^{\infty}(\xi) \rightarrow C^{\infty}(\eta)$ be of order (up to) $k$ and linear. We denote with $J^{k}(\xi)$ the corresponding $k$-jet bundle of $\xi$. For a local coordinate frame $\left(x^{\mu}, u^{a}\right)$ in $\xi$ there exists an induced coordinate frame in $J^{k}(\xi)$ denoted by $\left(x^{\mu}, u^{a}, u_{\mu}^{a}, \ldots, u_{\mu_{1} \mu_{2} \ldots \mu_{k}}^{a}\right)$, where the indices are ordered $\mu_{1} \leq \mu_{2} \leq \ldots \leq \mu_{k}$. Each vector $u \in J^{k}(\xi)_{x}$ is a jet of particular section $\psi: M \rightarrow E$, i.e. $u=j^{k}(\psi)_{x}$ so that

$$
u^{a}=\psi^{a}(x), \quad u_{\mu}^{a}=\partial_{\mu} \psi^{a}(x), \ldots, \quad u_{\mu_{1} \mu_{2} \ldots \mu_{k}}^{a}=\partial_{\mu_{1} \mu_{2} \ldots \mu_{k}} \psi^{a}(x) .
$$

Any linear differential operator is completely determined by its general symbol, i.e. the bundle morphism $\mathscr{D}: J^{k}(\xi) \rightarrow \eta$ (over the identity on $M$ ). Using some natural isomorphisms, one can view general symbols as sections in the tensor product $\left(J^{k}(\xi)\right)^{*} \otimes \eta$. The set of all linear differential operators of order up to $k$ corresponds one-to-one to smooth sections $C^{\infty}\left(\left(J^{k}(\xi)\right)^{*} \otimes \eta\right)$. The jet lifting of the sections $j^{k}: C^{\infty}(\xi) \rightarrow C^{\infty}\left(J^{k}(\xi)\right)$ plays the role of an universal differential operator of order (up to) $k$. An arbitrary linear differential operator $D: C^{\infty}(\xi) \rightarrow C^{\infty}(\eta)$ of order $k$ is a composition $D=\mathscr{D} \circ j^{k}$. If $D$ is a nonlinear operator then its general symbol is a fibre-preserving map $\mathscr{D}: J^{k}(\xi) \rightarrow \eta$. The action of the group $G$ in the bundle $\xi$ induces another action of $G$ in the 
corresponding jet bundle $J^{k}(\xi)$ (so-called jet lifting of the action). Thus we have an action of $G$ in the tensor product $\left(J^{k}(\xi)\right)^{*} \otimes \eta$. Then a differential operator is invariant if and only if its general symbol is an invariant section in $\left(J^{k}(\xi)\right)^{*} \otimes \eta$. So the jet bundle technique gives an algebraization of the (linear) differential operators. If we construct the reduced bundle of $\left(J^{k}(\xi)\right)^{*} \otimes \eta$ this will provide a full description of all invariant operators. In the nonlinear case general symbol mathscrD is a fibre-preserving map. At any point $x \in M$ the restriction $\mathscr{D}_{x}:\left(J^{k}(\xi)_{x} \rightarrow \eta_{x}\right)$ is a smooth (nonlinear) map. The description of the $G$-invariant nonlinear operators is similar to the linear case. We study the action of the stationary subgroup $H_{x_{0}}$ on the space $C^{\infty}\left(\left(J^{k}(\xi)\right)_{x_{0}}, \eta_{x_{0}}\right)-$ the space of all smooth maps $J^{k}(\xi)_{x_{0}} \rightarrow \eta_{x_{0}}$ and then we have to find the fixed elements in it. We use this scheme to describe all conformally invariant differential operators acting on Minkowski space. As a simple illustration we will consider the two dimensional algebraic conformal case. In both cases the base $M$ is a homogeneous space. The reduced bundle $\xi_{G}$ consists of one fibre over one point. The crucial point is to find the stationary elements of $C^{\infty}(\xi)_{x_{0}}$ for only one point.

\section{$3 \quad$ Illustrative example}

We consider the space of analytic functions of a single complex variable and the differential operators between analytic functions. The analytic functions may be viewed as sections on trivial line bundles over the complex plane $\mathbb{C}$, i.e. $\xi=(\mathbb{C} \times \mathbb{C}, p, \mathbb{C})$. The group $G L(2, \mathbb{C})$ acts on $\mathbb{C}$ by rational transformations

$$
t_{g}(z):=\frac{a z+b}{c z+d}, \quad z \in \mathbb{C}, \quad g=\left(\begin{array}{ll}
a & b \\
c & d
\end{array}\right) \in G L(2, \mathbb{C})
$$

$G L(2, \mathbb{C})$ acts on the analytic functions by transforming the argument. In adapted coordinates $(z, u)$ this action is

$$
g(z, u)=\left(\frac{a z+b}{c z+d}, u\right)
$$

The problem to solve is the description of all $G L(2, \mathbb{C})$-invariant differential operators. The invariance condition now reads

$$
D\left(f\left(\frac{a z+b}{c z+d}\right)\right)=(D(f))\left(\frac{a z+b}{c z+d}\right), \forall \quad g=\left(\begin{array}{ll}
a & b \\
c & d
\end{array}\right) \in G L(2, \mathbb{C}) .
$$

The complex plane is a homogeneous space, because translations in $\mathbb{C}$ are a subgroup of $G L(2, \mathbb{C})$

$$
g=\left(\begin{array}{ll}
1 & b \\
0 & 1
\end{array}\right) \quad \Longrightarrow \quad t_{g}(z)=z+b
$$

We choose $z_{0}=0$. Its stationary subgroup $H_{0}$ is defined as the subset

$$
H_{0}=\left\{h \in G L(2, \mathbb{C}) \mid h=\left(\begin{array}{ll}
a & 0 \\
c & 1
\end{array}\right) \quad a \neq 0\right\} .
$$

The fibre $J^{k}(\xi)_{0}$ is the set of all $k$-jets taken at $z=0$ of analytic functions. The $k$-jet of an analytic function is its Taylor expansion up to order $k$. If $w=j^{k}(f)_{0} \in J^{k}(\xi)_{0}$ then

$$
w=\sum_{l=1}^{k} \frac{1}{l !} u_{l} z^{l}
$$

where

$$
u_{l}=\frac{d^{l} f(0)}{d z^{l}}, \quad l=1,2, \ldots, k .
$$

We have assumed above for simplicity that $u_{0}=0$ since it doesn't lead to any loss of generality. 
The prolonged action of $H_{0}$ on the fibre $J^{k}(\xi)_{0}$ is given by the definition

$$
h(w)=j^{k}\left(f\left(\frac{a z}{c z+1}\right)\right)=\overline{j^{k}(f) \circ j^{k}\left(\frac{a z}{c z+1}\right)}, \quad h \in H_{0} .
$$

The bar over the right hand side of (11) indicates that the composition is truncated, i.e. all monomials of higher order than $k$ have been ignored. The jet of a composition of functions is the composition of their jets. On the other hand, the truncated Taylor expansion has the form

$$
j^{k}\left(\frac{a z}{c z+1}\right)=a z \sum_{m=0}^{k-1}(-c z)^{m} .
$$

The transformation $h\left(u_{1}, \ldots, u_{k}\right)=\left(w_{1}, \ldots, w_{k}\right)$ is determined by the equation

$$
\overline{\sum_{l=1}^{k} \frac{1}{l !} u_{l}\left(a z \sum_{m=0}^{k-1}(-c z)^{m}\right)^{l}}=\sum_{l=1}^{k} \frac{1}{l !} w_{l} z^{l}
$$

For example, the transformation of the jet of fourth order is

$$
\mid \begin{aligned}
& w_{1}=u_{1} a \\
& w_{2}=u_{2} a^{2}-2 u_{1} a c \\
& w_{3}=u_{3} a^{3}-6 u_{2} a^{2} c+6 u_{1} a c^{2} \\
& w_{4}=u_{4} a^{4}-12 u_{3} a^{3} c+36 u_{2} a^{2} c^{2}-24 u_{1} a c^{3} .
\end{aligned}
$$

Since we consider only scalar functions we have to describe maps $J^{k}(\xi)_{0} \rightarrow \mathbb{C}$ invariant under the action of $H_{0}$. Let $J^{k}(\xi)_{0} / H_{0}$ be the quotient space and $\pi: J^{k}(\xi)_{0} \rightarrow J^{k}(\xi)_{0} / H_{0}$ be the canonical projection on it. A map $\mathscr{D}_{0}: J^{k}(\xi)_{0} \rightarrow \mathbb{C}$ is said to be $H_{0}$-invariant if and only if there exists another map $\tilde{\mathscr{D}}_{0}: J^{k}(\xi)_{0} / H_{0} \rightarrow \mathbb{C}$ such that the relation $\mathscr{D}=\tilde{\mathscr{D}} \circ \pi$ holds. One may rewrite that requirement in terms of commutative diagrams as follows

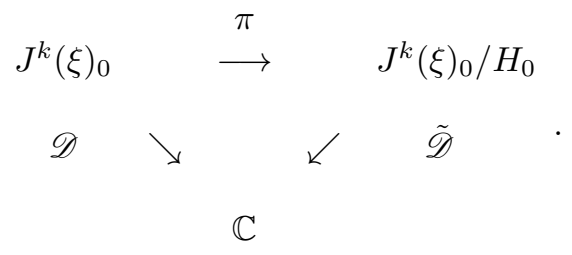

In this sense the canonical projection $\pi$ is a universal $H_{0}$-invariant map. The components of $\pi$ (in any coordinates in the quotient space) are invariant. Any $H_{0}$-invariant map is a function of the components of $\pi$.

To describe the quotient space means we must find a canonical representative in every orbit of $H_{0}$. The jets with $u_{1}=0$ is an invariant subspace. Considering the general case with $u_{1} \neq 0$ in each orbit there is an unique representative with $u_{1}=1$ and $u_{2}=0$. The projection on this canonical representative is given by the element $h \in H_{0}$ with $a=1 / u_{1}, c=u_{2} / 2 u_{1}$ more precisely

$$
\overline{\sum_{l=1}^{k} \frac{1}{l !} u_{l}\left(\frac{1}{u_{1}} z \sum_{m=0}^{k-1}\left(-\frac{u_{2}}{2 u_{1}} z\right)^{m}\right)^{l}}=z+\sum_{l=3}^{k} \frac{1}{l !} w_{l} z^{l} .
$$

The coefficients $w_{l}$ are coordinates in the quotient space

$$
\begin{gathered}
w_{3}=\frac{u_{3}}{\left(u_{1}\right)^{3}}-\frac{3}{2}\left(\frac{u_{2}}{\left(u_{1}\right)^{2}}\right)^{2} \\
w_{4}=\frac{u_{4}}{\left(u_{1}\right)^{4}}-6 \frac{u_{2} u_{3}}{\left(u_{1}\right)^{5}}+6 \frac{\left(u_{2}\right)^{3}}{\left(u_{1}\right)^{6}},
\end{gathered}
$$




$$
w_{5}=\frac{u_{5}}{\left(u_{1}\right)^{5}}-10 \frac{u_{2} u_{4}}{\left(u_{1}\right)^{6}}+30 \frac{\left(u_{2}\right)^{2} u_{3}}{\left(u_{1}\right)^{7}}-\frac{45}{2} \frac{\left(u_{2}\right)^{4}}{\left(u_{1}\right)^{8}}
$$

The coefficients $w_{l}$ are the general symbols of the $H_{0}$-invariant differential operators at $z=0$. Since translations act in a trivial manner on the $k$-jets they look exactly the same at any point of $\mathbb{C}$. If we consider the infinite order jets by using the previous method we can obtain the following generating power series

$$
\sum_{k=1}^{\infty} \frac{1}{k !} u_{k}\left(\frac{z}{u_{1}} \sum_{l=1}^{\infty}\left(-\frac{u_{2}}{2 u_{1}} z\right)^{l}\right)^{k}=z+\sum_{k=3}^{\infty} \frac{1}{k !} w_{k} z^{k} .
$$

The functions $w_{l}=w_{l}\left(u_{1}, u_{2}, \ldots, u_{l}\right), \quad l=3,4, \ldots$ are components of the canonical projection $\pi$. According to the previous remark the corresponding differential operators are universal (a complete system of invariants). Any invariant differential operator up to order $k$ is a function of them. The invariant differential operators corresponding to the symbols (17) and (18) look as follows

$$
D_{1}(f)=\frac{1}{\left(f^{\prime}(z)\right)^{2}}\left(\frac{f^{\prime \prime \prime}(z)}{f^{\prime}(z)}-\frac{3}{2}\left(\frac{f^{\prime \prime}(z)}{f^{\prime}(z)}\right)^{2}\right)
$$

and

$$
D_{2}(f)=\frac{f^{(I V)}(z)}{\left(f^{\prime}(z)\right)^{4}}-6 \frac{f^{\prime \prime \prime}(z) f^{\prime \prime}(z)}{\left(f^{\prime}(z)\right)^{5}}+6 \frac{\left(f^{\prime \prime}(z)\right)^{3}}{\left(f^{\prime}(z)\right)^{6}} .
$$

The expression in the parenthesis in (21) is the well-known Schwarz derivative. Thus we have confirmed the classic result that the Schwarz derivative is an invariant differential operator with respect to the action of fraction-linear transformations. The Schwarz derivative takes values in quadratic differentials. The difference between (21) and the Schwarz derivative is the factor $\left(f^{\prime}(z)\right)^{-2}$ because $D_{1}(f)$ takes values in scalar functions. Further more, we have obtained that there are $k-2$ functionally independent $G L(2, \mathbb{C})$-invariant differential operators of order $l \leq k$. Any invariant differential operator of the same order is a function of them.

Each invariant differential operator is nonlinear and it has a particular domain, i.e. it exists the requirement $f^{\prime}(z) \neq 0$.

Note: The fact that the $k$-jet $j^{k}(f)_{0}$ starts with 0 enables us to exclude the trivial case when the differential operators are operators of zero order, i.e. functions of $f$. It is clear that such operators are for sure $G L(2, \mathbb{C})$-invariant. Since we exclude the invariant subspace of jets with $u_{1}=0$ we obtain in general differential operators which involve division by a power of $f^{\prime}(z)$.

\section{Minkowski case}

Let us consider the Minkowski space $\mathscr{M}=\left(\mathbb{R}^{4}, \eta\right)$ with the pseudoeuclidean metric tensor $\eta=$ $\operatorname{diag}(-1,1,1,1)$. The conformal group $C(1,3)$ consists of all diffeomorphisms $\varphi: \mathbb{R}^{4} \rightarrow \mathbb{R}^{4}$ preserving the conformal class of $\eta$, i.e.

$$
\varphi^{*}(\eta)_{\mu \nu}(x)=\frac{\partial \varphi^{\alpha}}{\partial x^{\mu}}(x) \frac{\partial \varphi^{\beta}}{\partial x^{\nu}}(x) \eta_{\alpha \beta}(\varphi(x))=e^{w(x)} \eta_{\mu \nu}(x),
$$

where $w(x)$ is a smooth function( the Greek indices take the values $0,1,2,3)$. The conformal group $C(1,3)$ is induced by the following transformations

1. translations: $x^{\mu} \rightarrow x^{\mu}+a^{\mu}, \quad a \in \mathbb{R}^{4}$;

2. rotations: $x^{\mu} \rightarrow \Lambda_{\nu}^{\mu} x^{\nu}, \quad \Lambda_{\alpha}^{\mu} \eta_{\mu \nu} \Lambda_{\beta}^{\nu}=\eta_{\alpha \beta}$;

3. dilatation: $x^{\mu} \rightarrow \lambda x^{\mu}, \quad \lambda>0$;

4. special conformal transformations: $x^{\mu} \rightarrow\left(x^{\mu}+b^{\mu} x^{2}\right) /\left(1+2 x . b+x^{2} b^{2}\right), \quad b \in \mathbb{R}^{4}$. 
Note: The special conformal transformations have a correct global definition in the compactified Minkowski space.

The conformal group acts on the space of the smooth scalar functions $\mathbb{R}^{4} \rightarrow \mathbb{R}$ by transforming the argument. We are looking for differential operator between scalar functions invariant under the action of the conformal group. We are going to follow the scheme demonstrated in the precedent section.

The Minkowski space is a homogeneous space. The stationary subgroup $H_{0} \subset C(1,3)$ for $x=0$ is induced by the transformations

1. $x^{\mu} \rightarrow \Lambda_{\nu}^{\mu} x^{\nu}$

2. $x^{\mu} \rightarrow \lambda x^{\mu}$

3. $x^{\mu} \rightarrow\left(x^{\mu}+b^{\mu} x^{2}\right) /\left(1+2 x \cdot b+x^{2} b^{2}\right)$.

We must consider the action of $H_{0}$ on the jets $J^{k}\left(\mathbb{R}^{4}\right)_{0}=\left\{j^{k}(f)_{0} \mid f \in C^{\infty}(\mathbb{R})^{4}, f(0)=0\right\}$ and find the $H_{0}$-invariant functions $J^{k}\left(\mathbb{R}^{4}\right)_{0} \rightarrow \mathbb{R}$, i.e. we have to describe the canonical projection $\pi: J^{k}\left(\mathbb{R}^{4}\right)_{0} \rightarrow J^{k}\left(\mathbb{R}^{4}\right)_{0} / H_{0}$.

The first nontrivial case is $k=2$. The 2 -jet of a smooth function $f$ is the Taylor polynimial

$$
j^{2}(f)_{0}=u_{\alpha} x^{\alpha}+\frac{1}{2} u_{\alpha_{1} \alpha_{2}} x^{\alpha_{1}} x^{\alpha_{2}} .
$$

where $\left(u_{\alpha}, u_{\alpha_{1} \alpha_{2}}\right), \alpha_{1} \leq \alpha_{2}$ represent coordinates in the fibre $J^{k}\left(\mathbb{R}^{4}\right)_{0}$.

Let $\varphi: \mathbb{R}^{4} \rightarrow \mathbb{R}^{4}$ be a diffeomorphism with a fixed point $x=0$ then its second order jet at $x=0$ is

$$
\left(j^{2}(\varphi)_{0}\right)^{\mu}=A_{\nu}^{\mu} x^{\nu}+\frac{1}{2} A_{\nu_{1} \nu_{2}}^{\mu} x^{\nu_{1}} x^{\nu_{2}},
$$

assuming that $\operatorname{det}\left(A_{\nu}^{\mu}\right) \neq 0\left(A_{\nu_{1} \nu_{2}}^{\mu}\right.$ is arbitrary). The action of a diffeomorphism $\varphi$ on the space $J^{2}\left(\mathbb{R}^{4}\right)_{0}$ is given by

$$
\left(\begin{array}{c}
u_{\mu} \\
u_{\mu_{1} \mu_{2}}
\end{array}\right) \rightarrow\left(\begin{array}{c}
A_{\mu}^{\nu} u_{\nu} \\
A_{\mu_{1}}^{\nu_{1}} A_{\mu_{2}}^{\nu_{2}} u_{\nu_{1} \nu_{2}}+A_{\mu_{1} \mu_{2}}^{\nu} u_{\nu}
\end{array}\right)
$$

A special case is the action of the stationary subgroup $H_{0}$. We have for the prolonged action of the dilatation

$$
\left(\begin{array}{c}
u_{\mu} \\
u_{\mu_{1} \mu_{2}}
\end{array}\right) \rightarrow\left(\begin{array}{c}
\lambda u_{\mu} \\
\lambda^{2} u_{\mu_{1} \mu_{2}}
\end{array}\right)
$$

as well as for rotations

$$
\left(\begin{array}{c}
u_{\mu} \\
u_{\mu_{1} \mu_{2}}
\end{array}\right) \rightarrow\left(\begin{array}{c}
\Lambda_{\mu}^{\nu} u_{\nu} \\
\Lambda_{\mu_{1}}^{\nu_{1}} \Lambda_{\mu_{2}}^{\nu_{2}} u_{\nu_{1} \nu_{2}}
\end{array}\right)
$$

The infinite jet of special conformal transformations $\varphi$ reads

$$
\left(\left.j^{\infty}(\varphi(x))\right|_{0}\right)^{\mu}=\left(x^{\mu}+b^{\mu} x^{2}\right) \sum_{k=0}^{\infty}(-1)^{k}\left(2 b . x+b^{2} x^{2}\right)^{k},
$$

specifically for $k=2$

$$
\left(\left.j^{2}(\varphi(x))\right|_{0}\right)^{\mu}=x^{\mu}-2 x^{\mu} b \cdot x+b^{\mu} x^{2} .
$$

Thus the action is

$$
\left(\begin{array}{c}
u_{\mu} \\
u_{\mu_{1} \mu_{2}}
\end{array}\right) \rightarrow\left(\begin{array}{c}
u_{\mu} \\
u_{\mu_{1} \mu_{2}}-2 u_{\mu_{1}} b_{\mu_{2}}-2 u_{\mu_{2}} b_{\mu_{1}}+2 u_{\beta} b^{\beta} \eta_{\mu_{1} \mu_{2}}
\end{array}\right),
$$

where $b_{\mu}=\eta_{\mu \nu} b^{\nu}$. 
We will describe the quotient space $J^{2}\left(\mathbb{R}^{4}\right)_{0} / H_{0}$ by choosing a canonical representative from each orbit of $H_{0}$. Let $\left(u_{\alpha}, u_{\alpha_{1} \alpha_{2}}\right)$ be coordinates in $J^{2}\left(\mathbb{R}^{4}\right)_{0}$.

At this step we assume that $u^{2}:=\eta_{\mu \nu} u^{\mu} u^{\nu}<0$. By dilatation choosing $\lambda=1 / \sqrt{\left(-u^{2}\right)}$ we obtain another representative of the same equivalence class

$$
\left(\begin{array}{c}
u_{\alpha} \\
u_{\alpha_{1} \alpha_{2}}
\end{array}\right) \rightarrow\left(\begin{array}{c}
v_{\alpha} \\
v_{\alpha_{1} \alpha_{2}}
\end{array}\right)=\left(\begin{array}{c}
u_{\alpha} / \sqrt{-u^{2}} \\
u_{\alpha_{1} \alpha_{2}} /\left(-u^{2}\right)
\end{array}\right)
$$

By a special hyperbolic rotation

$$
A_{\alpha}^{\beta}=\left(\begin{array}{cc}
v_{0} & -\vec{v} \\
\vec{v} & \delta_{j}^{i}-v^{i} v_{j}\left(1+v_{0}\right) / \vec{v}^{2}
\end{array}\right),
$$

where we denote by $\vec{v}$ the space component $\left(v^{1}, v^{2}, v^{3}\right)$ of the 4 -vector $v$, we reach to another representative

$$
\left(\begin{array}{c}
v_{\alpha} \\
v_{\alpha_{1} \alpha_{2}}
\end{array}\right) \rightarrow\left(\begin{array}{c}
(1,0,0,0) \\
A_{\alpha_{1}}^{\beta_{1}} A_{\alpha_{2}}^{\beta_{2}} v_{\beta_{1} \beta_{2}}
\end{array}\right)=\left(\begin{array}{c}
e_{0} \\
w_{\alpha_{1} \alpha_{2}}
\end{array}\right)
$$

In the case of 2-jets looking like (31) the action (28) reads

$$
\mid \begin{array}{cl}
e_{0} & \rightarrow e_{0} \\
w_{0 \beta} & \rightarrow w_{0 \beta}-2 a_{\beta} \\
w_{i j} & \rightarrow w_{i j}-2 a_{0} \delta_{i j}
\end{array}
$$

This formula enables us to choose $a_{\beta}=w_{0 \beta} / 2$ and therefore we get another "more canonical" representative

$$
\left(\begin{array}{c}
e_{0} \\
\left(w_{\mu \nu}\right)
\end{array}\right) \rightarrow\left(\left(\begin{array}{cc}
(1,0,0,0) \\
0 & \overrightarrow{0} \\
\overrightarrow{0} & \tilde{w}_{m n}
\end{array}\right)\right)=:\left(\begin{array}{c}
e_{0} \\
w_{\alpha_{1} \alpha_{2}}
\end{array}\right) .
$$

Each equivalence class of this type of jets has got such a representative. The subgroup $O(3) \subset$ $C(1,3)$ is still acting on the jets (32) preserving their form. The action on symmetric $3 \times 3$ matrices is $\tilde{w} \rightarrow B \tilde{w} B^{T}, B \in O(3)$. That is why we can choose the transformation (a different boost) turning the matrix $\tilde{w}$ into a diagonal form

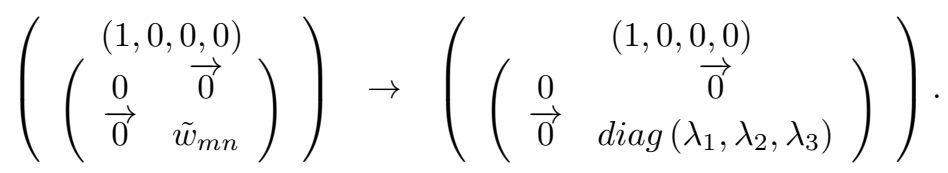

The unordered triple of eigenvalues $\left(\lambda_{1}, \lambda_{2}, \lambda_{3}\right)$ are coordinates in the quotient space $J^{2}\left(\mathbb{R}^{4}\right)_{0} / H_{0}$, so they describe completely this quotient space. As coordinate frame we may choose the elementary symmetric polynomials

$$
\sigma_{1}=\lambda_{1}+\lambda_{2}+\lambda_{3}, \quad \sigma_{2}=\lambda_{1} \lambda_{2}+\lambda_{1} \lambda_{3}+\lambda_{2} \lambda_{3}, \quad \sigma_{3}=\lambda_{1} \lambda_{2} \lambda_{3}
$$

but we prefer working with the following frame

$$
\begin{aligned}
& S_{1}\left(\lambda_{1}, \lambda_{2}, \lambda_{3}\right)=\lambda_{1}+\lambda_{2}+\lambda_{3} \\
& S_{2}\left(\lambda_{1}, \lambda_{2}, \lambda_{3}\right)=\left(\lambda_{1}\right)^{2}+\left(\lambda_{2}\right)^{2}+\left(\lambda_{3}\right)^{2} \\
& S_{3}\left(\lambda_{1}, \lambda_{2}, \lambda_{3}\right)=\left(\lambda_{1}\right)^{3}+\left(\lambda_{2}\right)^{3}+\left(\lambda_{3}\right)^{3} .
\end{aligned}
$$

because of its convenient form

$$
S_{k}\left(\lambda_{1}, \lambda_{2}, \lambda_{3}\right)=\operatorname{Tr}\left(\tilde{w}^{k}\right), \quad k=1,2,3 .
$$


The quantities $S_{1}, S_{2}$ and $S_{3}$ are coordinates in the equivalence class of the jet that we started from. To obtain them as explicit functions of the initial jet we must take the composition

$$
\left(\begin{array}{c}
u_{\alpha} \\
u_{\alpha_{1} \alpha_{2}}
\end{array}\right) \rightarrow\left(\begin{array}{c}
v_{\alpha} \\
v_{\alpha_{1} \alpha_{2}}
\end{array}\right) \rightarrow\left(\begin{array}{c}
e_{0} \\
w_{\alpha_{1} \alpha_{2}}
\end{array}\right) \rightarrow\left(\begin{array}{c}
e_{0} \\
\tilde{w}_{\alpha_{1} \alpha_{2}}
\end{array}\right) \rightarrow\left(\begin{array}{c}
\operatorname{Tr}(\tilde{w}) \\
\operatorname{Tr}(\tilde{w})^{2} \\
\operatorname{Tr}(\tilde{w})^{3}
\end{array}\right)
$$

The functions $\mathscr{D}_{k}=\operatorname{Tr}\left(\tilde{w}^{k}\right)=\mathscr{D}_{k}\left(u_{\alpha}, u_{\alpha_{1} \alpha_{2}}\right)$ are the general symbols of the invariant operators at $x=0$. Since the translations act trivially on the jets the differential operators look in the same way at any point of the Minkowski space. The final result is

$$
\begin{gathered}
D_{1}(f)=\frac{\nabla^{2} f}{(\nabla f)^{2}}+2 \frac{\partial^{\alpha} f \partial^{\beta} f}{(\nabla f)^{2}} \frac{\partial_{\alpha \beta} f}{(\nabla f)^{2}} . \\
D_{2}(f)=\left(\eta^{\alpha \mu} \eta^{\beta \lambda}-2 \eta^{\alpha \mu} \frac{\partial^{\beta} f \partial^{\lambda} f}{(\nabla f)^{2}}+2 \frac{\partial^{\alpha} f \partial^{\beta} f}{(\nabla f)^{2}} \frac{\partial^{\lambda} f}{(\nabla f)^{2}}\right) \frac{\partial_{\alpha \beta} f}{(\nabla f)^{2}} \frac{\partial_{\lambda \mu} f}{(\nabla f)^{2}}+ \\
2 \frac{\nabla^{2} f}{(\nabla f)^{2}} \frac{\partial^{\alpha} f \partial^{\beta} f}{(\nabla f)^{2}} \frac{\partial_{\alpha \beta} f}{(\nabla f)^{2}} \\
D_{3}(f)=C^{\alpha \beta \lambda \mu \rho \sigma} \frac{\partial_{\alpha \beta} f}{(\nabla f)^{2}} \frac{\partial_{\lambda \mu} f}{(\nabla f)^{2}} \frac{\partial_{\rho \sigma} f}{(\nabla f)^{2}}+3 \frac{\nabla^{2} f}{(\nabla f)^{2}} \frac{\partial^{\alpha} f \partial^{\beta} f}{(\nabla f)^{2}} \frac{\partial^{\lambda} f \partial^{\mu} f}{(\nabla f)^{2}} \frac{\partial_{\alpha \beta} f}{(\nabla f)^{2}} \frac{\partial_{\lambda \mu} f}{(\nabla f)^{2}},
\end{gathered}
$$

where

$$
\begin{aligned}
C^{\alpha \beta \lambda \mu \rho \sigma}:= & \eta^{\alpha \sigma} \eta^{\beta \lambda} \eta^{\mu \rho}-3 \eta^{\alpha \sigma} \eta^{\beta \lambda} \frac{\partial^{\mu} f \partial^{\rho} f}{(\nabla f)^{2}}+3 \eta^{\alpha \mu} \eta^{\beta \lambda} \frac{\partial^{\rho} f \partial^{\sigma} f}{(\nabla f)^{2}}+ \\
& 3 \eta^{\alpha \sigma} \frac{\partial^{\beta} f \partial^{\lambda} f}{(\nabla f)^{2}} \frac{\partial^{\mu} f \partial^{\rho} f}{(\nabla f)^{2}}-6 \eta^{\alpha \mu} \frac{\partial^{\beta} f \partial^{\lambda} f}{(\nabla f)^{2}} \frac{\partial^{\rho} f \partial^{\sigma} f}{(\nabla f)^{2}}+ \\
& 2 \frac{\partial^{\alpha} f \partial^{\beta} f}{(\nabla f)^{2}} \frac{\partial^{\lambda} f \partial^{\mu} f}{(\nabla f)^{2}} \frac{\partial^{\rho} f \partial^{\sigma} f}{(\nabla f)^{2}}, \\
& (\nabla f)^{2}=\eta^{\alpha \beta} \partial_{\alpha} f \partial_{\beta} f, \quad \nabla^{2} f=\eta^{\alpha \beta} \partial_{\alpha \beta} f
\end{aligned}
$$

So we have just obtained the differential operators having considered functions with time-like gradients. According to the general scheme these differential operators are functionally independent and universal, i.e. they generate all conformally invariant operators of second order (defined on functions with time-like gradient). This procedure also contains an algorithm for calculating the higher order differential operators (defined on the same subset of functions).

The natural framework of describing the conformally invariant differential operators involves the complexified and compactified Minkowski space.

This technique is applicable to the case of $n$-dimensional pseudoeuclidean space too. There are no conformally invariant first order differential operators. The conformally invariant differential operators of second order are generated by $n-1$ functionally independent differential invariants. For example, one of these invariant operators (involving functions with time-like gradient) is the following one

$$
D(f)=\frac{\nabla^{2} f}{(\nabla f)^{2}}+(n-2) \frac{\partial^{\alpha} f \partial^{\beta} f}{(\nabla f)^{2}} \frac{\partial_{\alpha \beta} f}{(\nabla f)^{2}} \quad \alpha, \beta=1 \ldots n
$$

\section{Acknowledgement.}

That study is supported by the contract 35/2003 with Sofia University. 


\section{References}

[1] Arnold, V.I., Varchenko, A.N., Hussein-Zade, S.M. Singularities of Smooth Mappinrgs I, II Nauka Publ., Moscow, 1982 (in Russian)

[2] Bröcker, Th. Differential Germs and Catastrophes Cambridge Univ. Press, 1975

[3] Saunders, D.J., The Geometry of Jet Bundles., Cambridge Univ. Press, Cambridge, 1989

[4] Palais, R.S., Seminar on Atiyah-Singer Index Theorem Princeton Univ. Press, Princeton, 1965

[5] Olver, P.J., Equivalence, Invatiants and Symmetry., Cambridge Univ. Press, New York, 1995

[6] Botcharov, A.V., Verbovetsky, A.M., Vinogradov, A.M. etc., Symmetries and Conservation Laws in The Equations of Mathematical Physics., Factorial Publ., Moscow, 1997 (in Russian) 